\title{
ON THE MAPPING TORUS OF AN AUTOMORPHISM
}

\author{
WILLIAM L. PASCHKE ${ }^{1}$
}

\begin{abstract}
Let $\rho$ be an automorphism of a $C^{*}$-algebra $A$. The mapping torus $T_{\rho}(A)$ is the $C^{*}$-algebra of $A$-valued continuous functions $x$ on $[0,1]$ satisfying $x(1)=$ $\rho(x(0))$. Using his Thom isomorphism theorem. A. Connes has shown that the $K$-groups of $T_{p}(A)$, with indices reversed, are isomorphic to those of the crossed product $A \times{ }_{p} Z$. We provide here an alternative proof of this fact which gives an explicit description of the isomorphism.
\end{abstract}

Given a $C^{*}$-algebra $A$ and an automorphism $\rho$ of $A$, the mapping torus of the pair $(A, \rho)$ is defined by

$$
T_{\rho}(A)=\{x \in C(I, A): x(1)=\rho(x(0))\},
$$

where $I$ is the unit interval and $C(I, A)$ is the $C^{*}$-algebra of continuous $A$-valued functions on $I$. In [1], A. Connes shows that the $K$-groups, with indices reversed, of $T_{\rho}(A)$ coincide with those of $A \times{ }_{\rho} Z$ (the crossed product of $A$ by the action of $Z$ generated by $\rho$ ). He does this in order to obtain the exact sequence of M. Pimsner and D. Voiculescu [3] for $K_{\#}\left(A \times_{\rho} Z\right)$ as a consequence of the Thom isomorphism in [1]. In the present note, we give an alternative proof of the isomorphism of $K_{1-j}\left(T_{\rho}(A)\right)$ with $K_{j}\left(A \times_{\rho} Z\right)$ which proceeds in somewhat the reverse fashion, namely starting from the sequence in [3]. One merit of this approach is that the isomorphism in question is described quite explicitly in terms of the elementary ingredients of $K$-theory.

We begin by recalling some pertinent facts and establishing notation. The crossed product $A \times{ }_{\rho} Z$ is generated by terms of the form $a L^{n}(a \in A, n \in Z)$, where $L=L_{\rho}$ is a unitary on the space of a certain representation of $A$ satisfying $L a L^{*}=\rho(a) \forall a \in A$. It will be convenient to assume that $A$ is unital throughout most of what follows, a restriction that is easily removed at the appropriate time. For such $A$, we have $L \in A \times{ }_{\rho} Z$. The exact sequence

$$
\begin{array}{ccccc}
K_{0}(A) & \stackrel{\rho_{*}-\text { id }}{\rightarrow} & K_{0}(A) & \stackrel{i_{*}}{\rightarrow} & K_{0}\left(A \times_{\rho} Z\right) \\
\uparrow & & & & \downarrow \\
K_{1}\left(A \times_{\rho} Z\right) & \stackrel{i_{*}}{\leftarrow} & K_{1}(A) & \stackrel{\rho_{*} \text {-id }}{\leftarrow} & K_{1}(A)
\end{array}
$$

Received by the editors September 2, 1982.

1980 Mathematics Subject Classification. Primary 46L05.

'Research partially supported by NSF grant MCS-8002138. 
was first obtained in [3] and subsequently in [2] and [1]. Here $i: A \rightarrow A \times_{\rho} Z$ is the natural inclusion, and the vertical arrows represent boundary maps to be discussed presently. In [3], the sequence above comes from an extension

$$
0 \rightarrow A \otimes \mathscr{K} \rightarrow \mathcal{T} \rightarrow A \times{ }_{\rho} Z \rightarrow 0
$$

of $A \times{ }_{\rho} Z$, called the Toeplitz extension. To construct $\mathcal{T}$, represent $A \times{ }_{\rho} Z$ on a Hilbert space $H$, and let $U_{+}$be the unilateral shift on $l^{2}\left(Z^{+}\right)$. The Toeplitz algebra $\mathcal{T}$ is then the $C^{*}$-algebra on $H \otimes l^{2}\left(Z^{+}\right)$generated by $A \otimes 1$ and the isometry $L \otimes U_{+}$. It is shown in [3] that there is a homomorphism $\pi: \sigma \rightarrow A \times{ }_{\rho} Z$, taking $a \otimes 1$ to $a$ and $L \otimes U_{+}$to $L$, whose kernel is isomorphic to $A \otimes \mathcal{K}$. Most of the hard work in [3] consists in proving that the injection $a \mapsto a \otimes 1$ of $A$ into $T$ induces an isomorphism of $K$-groups. Identifying $K_{\#}(\mathcal{T})$ with $K_{\#}(A)$ in this manner transforms the $K$-theory exact sequence ( $\S 10$ of [4]) for (2) into the sequence (1). In particular, if $u$ is a unitary in $A \times{ }_{\rho} Z$ that lifts to a partial isometry $V$ in $T$, the boundary map $\partial: K_{1}\left(A \times_{\rho} Z\right) \rightarrow K_{0}(A)$ takes the class of $u$ to the index of $V$ computed in $K_{0}(A \otimes \mathcal{K}) \approx K_{0}(A)$.

Consider now the map $e: T_{\rho}(A) \rightarrow A$ defined by $e(x)=x(0)$. The kernel of $e$ is the reduced suspension $S A(=\{x \in C(I, A): x(0)=0=x(1)\})$, so, via the Bott maps that identify $K_{j}(S A)$ with $K_{1-j}(A)$, we have an exact sequence

$$
\begin{array}{ccccc}
K_{1}(A) & \rightarrow & K_{0}\left(T_{\rho}(A)\right) & \stackrel{e}{\rightarrow} & K_{0}(A) \\
\uparrow & & & & \downarrow \\
K_{1}(A) & \underset{e_{*}}{\leftarrow} & K_{1}\left(T_{\rho}(A)\right) & \leftarrow & K_{0}(A)
\end{array}
$$

It is the following lemma that suggests a relationship between (3) and (1).

LEMMA 1. The vertical arrows in (3) both represent $\rho_{*}-\mathrm{id}$.

Proof. There is no loss of generality in working with unitaries and projections in $A$ rather than in $A \otimes M_{n}$, since $T_{\rho \otimes \text { id }_{n}}\left(A \otimes M_{n}\right)$ is isomorphic to $T_{\rho}(A) \otimes M_{n}$. For the $K_{0}$ arrow, let $p$ be a projection on $A$ and [ $p$ ] its class in $K_{0}(A)$. Define $x \in T_{\rho}(A)$ by $x(t)=(1-t) p+t \rho(p)$, so $e(x)=p$. The map $\delta: K_{0}(A) \rightarrow K_{1}(S A)$ from $S A \rightarrow T_{\rho}(A) \rightarrow A$ then takes $[p]$ to $\left[e^{2 \pi i x}\right] \in K_{1}\left(S A^{+}\right)=K_{1}(S A)$ (where ${ }^{+}$denotes adjunction of a unit). But if $\beta: K_{0}(A) \rightarrow K_{1}(S A)$ is the Bott map, we also have $\left[e^{2 \pi i x}\right]=\beta[\rho(p)]-\beta[p]$. This takes care of the $K_{0}$ case. One can give a similar, but somewhat more cumbersome, direct argument for $K_{1}$, or else proceed by replacing $A$ with $A \otimes C(T), \rho$ with $\tilde{\rho}=\rho \otimes \mathrm{id}_{T}$, and identifying $T_{\tilde{\rho}}(A \otimes C(T))$ with $T_{\rho}(A) \otimes C(T)$. Write $\nu: K_{\mathrm{l}}(A) \rightarrow K_{1}(A)$ for the left-hand vertical arrow in (3). When we decompose $K_{0}(A \otimes C(T))$ as $K_{0}(A) \oplus K_{1}(A)$, the right-hand arrow in the $A \otimes C(T)$ version of (3) is, on the one hand, $\left(\rho_{*}-\mathrm{id}\right) \oplus \nu$, and on the other, $\tilde{\rho}_{*}-\mathrm{id}=\left(\rho_{*}-\mathrm{id}\right) \oplus\left(\rho_{*}-\mathrm{id}\right)$, so $\nu=\rho_{*}-\mathrm{id}$. 
By the lemma, we have two parallel exact sequences:

$$
\begin{aligned}
& K_{0}\left(T_{\rho}(A)\right) \\
& \alpha_{\nearrow} \stackrel{e}{ }^{*} \\
& \rightarrow K_{1}(A) \underset{\rho_{*}-\text { id }}{\rightarrow} K_{1}(A) \\
& i_{*} \searrow \\
& K_{0}(A) \underset{\rho_{*} \rightarrow \text { id }}{\rightarrow} K_{0}(A) \rightarrow \\
& K_{1}\left(A \times_{\rho} Z\right)
\end{aligned}
$$

where $\alpha$ is the composition of the Bott map $K_{1}(A) \rightarrow K_{0}(S A)$ with the map induced by the inclusion of $S A$ into $T_{\rho}(A)$. What we need is a homomorphism $\gamma: K_{0}\left(T_{\rho} A\right) \rightarrow$ $K_{1}\left(A \times{ }_{\rho} Z\right)$ satisfying $\gamma \alpha=i_{*}$ and $\partial \gamma=e_{*}$. Such a map will automatically be an isomorphism by exactness of the two sequences.

The following lemma, needed in the definition of $\gamma$, is a variant of the familiar fact that norm-close projections in a unital $C^{*}$-algebra are unitarily equivalent.

Lemma 2. Let $\left\{p_{t}: t \in I\right\}$ be a path of projections in $A$. There is a path $\left\{w_{t}\right\}$ of unitaries in $A$ with $w_{0}=1$ and $p_{t}=w_{t} p_{0} w_{t}^{*} \forall t \in I$.

Proof. Fix $t_{0} \in I$. There is an $\varepsilon>0$ (independent of $t_{0}$ ) such that whenever $\left|t-t_{0}\right|<\varepsilon$, we have $\left\|p_{t}-p_{t_{0}}\right\|<1$ and $\left\|r_{t}-p_{t}\right\|<1$, where $r_{t}$ is the idempotent $\left(1-p_{t_{0}}+p_{t}\right)^{-1} p_{t_{0}}\left(1-p_{t_{0}}+p_{t}\right)$. Notice that for such $t$, we have $r_{t}=r_{t} p_{t}$. Let $x_{t}=$ $\left(1-p_{t}+r_{t}\right)^{-1}\left(1-p_{t_{0}}+p_{t}\right)^{-1}$. Then, easily, $x_{t}^{-1} p_{t}=p_{t_{0}} x_{t}^{-1}$ for $t$ within $\varepsilon$ of $t_{0}$. Partitioning $I$ into subintervals of length less than $\varepsilon$, we can chain together the partial paths $\left\{x_{t}\right\}$ to obtain a full path $\left\{y_{t}\right\}$ of invertibles, with $y_{0}=1$, such that $p_{t}=y_{t} p_{0} y_{t}^{-1}$. Since $\left|y_{t}\right|$ commutes with $p_{0}$ for each $t$, we may replace $\left\{y_{t}\right\}$ by the unitary path $\left\{w_{t}\right\}=\left\{y_{t}\left|y_{t}\right|^{-1}\right\}$.

Now we can move on to the main result.

TheOREM (CONNES [1]). For a $C^{*}$-algebra $A$ and an automorphism $\rho$ of $A$, the groups $K_{j}\left(A \times_{\rho} Z\right)$ and $K_{1-j}\left(T_{\rho}(A)\right)$ are isomorphic $(j=0,1)$.

Proof. We will assume that $A$ is unital for most of the argument, and deal directly just with the case $j=1$. (The other isomorphism will be described more or less concretely in a separate remark.) Let $p$ be a projection in $T_{\rho}(A)$ and let $\left\{w_{t}\right\}$ be an implementing path for $\{p(t)\}$ as in Lemma 2. In $A \times_{\rho} Z$ we have $L p(0) L^{*}=$ $\rho(p(0))=p(1)=w_{1} p(0) w_{1}^{*}$, so $L^{*} w_{1}$ commutes with $p(0)$. Thus $L^{*} w_{1} p(0)+1-$ $p(0)$, which we shall denote temporarily by $\gamma_{0}(p)$, is a unitary in $A \times_{\rho} Z$. If $\left\{v_{t}\right\}$ is another implementing path for $\{p(t)\}$, then $w_{t}^{*} v_{t}$ commutes with $p(0)$ for each $t$ and $t \mapsto L^{*} w_{1} w_{t}^{*} v_{t} p(0)+1-p(0)$ is a path of unitaries in $A \times{ }_{\rho} Z$ joining $L^{*} w_{1} p(0)+1$ $-p(0)$ to $L^{*} v_{1} p(0)+1-p(0)$. Thus the class $\left[\gamma_{0}(p)\right]$ in $K_{1}\left(A \times_{\rho} Z\right)$ is independent of the choice of implementing path. Further, if $q$ is a projection in $T_{\rho}(A)$ unitarily equivalent to $p$, say $q=u p u^{*}$ for some unitary $u \in T_{\rho}(A)$, then 
$\left\{u(t) w_{t} u(0)^{*}\right\}$ is an implementing path for $\{q(t)\}$. In $K_{1}\left(A \times{ }_{\rho} Z\right)$ we have

$$
\begin{aligned}
{\left[\gamma_{0}(q)\right] } & =\left[L^{*} u(1) w_{1} u(0)^{*} q(0)+1-q(0)\right] \\
& =\left[L^{*} \rho\left(u(0)^{*}\right) u(1) w_{1} p(0)+1-p(0)\right]=\left[\gamma_{0}(p)\right]
\end{aligned}
$$

(conjugating by $u(0)^{*}$ and using $\rho(u(0))=u(1)=L u(0) L^{*}$ ). Replacing $A$ by $A \otimes M_{n}$ and $\rho$ by $\rho \otimes \mathrm{id}_{n}$, we can define $\gamma_{0}(p)$ in $\left(A \times_{\rho} Z\right) \otimes M_{n}$ for a projection $p$ in $A \otimes M_{n}$. Set $\gamma[p]=\left[\gamma_{0}(p)\right]$. The resulting map $\gamma: K_{0}\left(T_{\rho}(A)\right) \rightarrow K_{1}\left(A \times_{\rho} Z\right)$ is well defined by what has been observed above and obviously a homomorphism. We next check that $\gamma \alpha=i_{*}$. Let $u$ be a unitary in (without loss of generality) $A$ and let $\left\{v_{t}\right\}$ be a path of unitaries in $A \otimes M_{2}$ with $v_{0}=1 \oplus 1$ and $v_{1}=u \oplus u^{*}$. Define $p \in$ $T_{\rho}(A) \otimes M_{2}$ by $p(t)=v_{t}(1 \oplus 0) v_{t}^{*}$. Then $\alpha[u]=[p]-[1]$ (see $\S 8$ of [4]) and

$$
\gamma([p])=\left[\left(L^{*} \oplus L^{*}\right)\left(u \oplus u^{*}\right)(1 \oplus 0)+(0 \oplus 1)\right]=\left[L^{*} u\right] .
$$

Since $\gamma[1]=\left[L^{*}\right]$, we have $\gamma \alpha[u]=i_{*}[u]$. To see that $\partial \gamma=e_{*}$, let $p$ be a projection in $T_{\rho}(A)$, with $\left\{w_{t}\right\}$ an implementing path for $\{p(t)\}$. We lift $\gamma_{0}(p)$ to $V \in \tau$, defined by

$$
V=\left(L^{*} \otimes U_{+}^{*}\right)\left(w_{1} \otimes 1\right)(p(0) \otimes 1)+(1-p(0)) \otimes 1
$$

We have

$$
V^{*} V=p(0) \otimes U_{+} U_{+}^{*}+(1-p(0)) \otimes 1=1 \otimes 1-p(0) \otimes\left(1-U_{+} U_{+}^{*}\right),
$$

and $V V^{*}=1 \otimes 1$, so $\partial\left[\gamma_{0}(p)\right]=[p(0)]=e_{*}[p]$ as required. It follows that $\gamma$ is an isomorphism.

Suppose now that $A$ is not unital. Adjoining a unit, we obtain $A^{+}$and its automorphism $\rho^{+}$. The exact sequence $0 \rightarrow A \rightarrow A^{+} \rightarrow \mathbf{C} \rightarrow 0$ gives rise to exact sequences

$$
\begin{aligned}
& 0 \rightarrow T_{\rho}(A) \rightarrow T_{\rho}\left(A^{+}\right) \rightarrow C(T) \rightarrow 0, \\
& 0 \rightarrow A \times{ }_{\rho} Z \rightarrow A^{+} \times_{\rho^{+}} Z \rightarrow C(T) \rightarrow 0
\end{aligned}
$$

in which the maps onto $C(T)$ admit right inverses. There are thus natural isomorphisms

$$
K_{0}\left(T_{\rho}\left(A^{+}\right)\right) \approx K_{0}\left(T_{\rho}(A)\right) \oplus Z, \quad K_{1}\left(A^{+} \times_{\rho^{+}} Z\right) \approx K_{1}\left(A \times_{\rho} Z\right) \oplus Z .
$$

The isomorphism $\gamma^{+}: K_{0}\left(T_{\rho}\left(A^{+}\right)\right) \rightarrow K_{1}\left(A^{+} \times_{\rho^{+}} Z\right)$ takes [1] to [ $L_{\rho^{+}}^{*}$, so it respects these direct sum decompositions and restricts to an isomorphism of $K_{0}\left(T_{\rho}(A)\right)$ with $K_{1}\left(A \times{ }_{\rho} Z\right)$.

Finally, replacing $A$ by $A \otimes C(T)$ and $\rho$ by $\rho \otimes \mathrm{id}_{T}$, we have the isomorphism $\tilde{\gamma}$ : $K_{0}\left(T_{\rho}(A) \otimes C(T)\right) \rightarrow K_{1}\left(\left(A \times_{\rho} Z\right) \otimes C(T)\right)$. If $\lambda$ and $\mu$ denote the natural inclusions of $T_{\rho}(A)$ and $A \times{ }_{\rho} Z$ into their tensor products with $C(T)$, it is clear that $\tilde{\gamma} \lambda_{*}=\mu_{*} \gamma$. Thus, when we decompose $K_{0}\left(T_{\rho}(A) \otimes C(T)\right)$ as $K_{0}\left(T_{\rho}(A)\right) \oplus K_{1}\left(T_{\rho}(A)\right)$, and likewise for $K_{1}\left(\left(A \times_{\rho} Z\right) \otimes C(T)\right)$, $\tilde{\gamma}$ maps $K_{0}\left(T_{\rho}(A)\right)$ isomorphically onto $K_{1}\left(A \times{ }_{\rho} Z\right)$. Hence the remaining direct summands must be isomorphic; the restriction of $\tilde{\gamma}$ to $K_{1}\left(T_{\rho}(A)\right)$ is the desired $K_{1}$-to- $K_{0}$ map. 
In general, $\rho_{*}$ and $K_{\#}(A)$ do not determine $K_{\#}\left(A \times_{\rho} Z\right)$ unambiguously. However, an easy consequence of the theorem is that $K_{\#}\left(A \times_{\rho} Z\right)$ depends on $\rho$ only up to homotopy.

CoROLlary. Suppose that $\rho_{0}$ and $\rho_{1}$ are automorphisms of $A$ joined by a (point-norm continuous $)$ path $\left\{\rho_{t}\right\}$ of automorphisms. Then $K_{\#}\left(A \times_{\rho_{0}} Z\right)$ and $K_{\#}\left(A \times{ }_{\rho_{1}} Z\right)$ are isomorphic.

Proof. For $x \in T_{\rho_{0}}(A)$ define $\theta x: I \rightarrow A$ by $(\theta x)(t)=\rho_{t} \rho_{0}^{-1}(x(t))$. The inequality

$$
\|(\theta x)(t)-(\theta x)(s)\| \leqslant\left\|\rho_{t} \rho_{0}^{-1}(x(t))-\rho_{s} \rho_{0}^{-1}(x(t))\right\|+\|x(t)-x(s)\|
$$

shows that $\theta x$ is continuous, and we have $(\theta x)(0)=x(0),(\theta x)(1)=\rho_{1} \rho_{0}^{-1}(x(1))=$ $\rho_{1}((\theta x)(0))$. Thus, $\theta$ is an isomorphism of $T_{\rho_{0}}(A)$ with $T_{\rho_{1}}(A)$.

In conclusion, we remark that an isomorphism of $K_{1}\left(T_{\rho}(A)\right)$ with $K_{0}\left(A \times{ }_{\rho} Z\right)$ can be defined formulaically as follows. (For simplicity assume that $A$ is unital.) Let $H$ be a selfadjoint $2 \times 2$ scalar matrix with $e^{i H}=\left(\begin{array}{ll}0 & 1 \\ 1 & 0\end{array}\right)$. Given a unitary $u \in T_{\rho}(A)$, define a unitary $V_{u} \in S\left(A \times_{\rho} Z\right)^{+} \otimes M_{2}$ by

$$
V_{u}(t)=(1 \oplus L) e^{i t H}\left(\rho^{-1}(u(1-t))^{*} \oplus 1\right) e^{-i t H}\left(1 \oplus L^{*}\right) e^{i t H}(u(0) \oplus 1) e^{-i t H}
$$

and analogously for unitaries in $T_{\rho}(A) \otimes M_{n}$. It is clear that $[u] \rightarrow\left[V_{u}\right]$ defines a homomorphism from $K_{1}\left(T_{\rho}(A)\right)$ to $K_{1}\left(S\left(A \times_{\rho} Z\right)\right)$. Composition of this with the Bott isomorphism $K_{1}\left(S\left(A \times{ }_{\rho} Z\right)\right) \rightarrow K_{0}\left(A \times_{\rho} Z\right)$ gives a homomorphism which can be shown to compose correctly with the appropriate maps in the index-reversed version of (4) and is thus an isomorphism. We omit details.

\section{REFERENCES}

1. A. Connes, An analogue of the Thom isomorphism for crossed products of a $C^{*}$-algebra by an action of $R$, Advances in Math. 39 (1981), 31-55.

2. J. Cuntz, $K$-theory for certain $C^{*}$-algebras. II, J. Operator Theory 5 (1981), 101-108.

3. M. Pimsner and D. Voiculescu, Exact sequences for $K$-groups and Ext-groups of certain cross-products $C^{*}$-algebras, J. Operator Theory 4 (1980), 93-118.

4. J. Taylor, Banach algebras and topology, Algebras in Analysis (J. Williamson, editor), Academic Press, New York, 1975.

Department of Mathematics, University of Kansas, Lawrence, Kansas 66045 